\title{
Assessment of Thermal Performance of Non-Conventional Grooved Stepped Shoe Ribs by CFD Technique
}

\author{
Sameer Y. Bhosale, G. R. Selokar
}

\begin{abstract}
In improvement of the thermal performance there is necessity of the heat transfer augmentation. Heat transfer enhancement can be achieved with enlarged or extended surface, impeded boundary level, augmentation in the turbulence etc. It is desired to keep the size of heat exchanger compact for better working conditions. In the proposed work, we made the Computational Fluid Dynamics (CFD) analysis of the non-conventional type of ribs. In this work the non-conventional Stepped grooved shoe shaped ribs were studied by changing its geometry parameters like rib height $(15,20,22 \mathrm{~mm})$, thickness of the rib $(4,5,10 \mathrm{~mm})$, and the ratio between these entities. The numerical analysis was done to study change in rate of heat transfer and pressure drop. The effects of variation in staggered arrangements and truncation gap on thermal performance were also studied. It was observed that providing staggered arrangement with truncation gap of $20 \mathrm{~mm}$ gives the optimum value of thermal enhancement factor of 1.33 .
\end{abstract}

Keywords: Modified shoe shape, stepped shoe shape rib, heat transfer enhancement, thermal enhancement

\section{INTRODUCTION}

Now a days it very important to augment the heat transfer as it applies to heavy industries to electronic components. Hence many researches have been done for the improvement of the heat transfer enhancement techniques. The shapes like the ribs, pin, fins and dimples are introduced to develop turbulence on the heat transmitting area. Ribs on the flat plate impede the thermal boundary layer development and it gives the boost to the turbulent kinetic energy and which leads to the augmented turbulent heat transferpaper are fine and satisfactory. Author (s) can make rectification in the final paper but after the final submission to the journal, rectification is not possible.Deep Singh Thakur, et.al, [1] The rib arrays and geometry have been studied by performing simulation runs by comparing the height $0.5 \mathrm{~mm}$ to $2 \mathrm{~mm}$, with pitch $10 \mathrm{~mm}$ to $20 \mathrm{~mm}$. The optimum results are achieved for $\mathrm{e}=1 \mathrm{~mm}$ and $\mathrm{P}=10 \mathrm{~mm}$ at $\mathrm{Re}=6000$. This rib is evaluated with the comparison of triangular, rectangular and semicircular rib shapes and it is seen that this rib gives

Revised Manuscript Received on December 30, 2019.

* Correspondence Author

Mr. Sameer Y. Bhosale* Sri SatyaSai University of Technology \& Medical Sciences Opposite Oilfed Plant, Indore-Bhopal Road,Sehore (Madhya Pradesh), Pin - 466001/Department of Mechanical Engineering, PES's Modern College of Engineering, 1186/A, Off J.M. Rd, Shivajinagar, Pune , Maharashtra,Pin- 411005

Prof. Dr. G. R. Selokar, Sri SatyaSai University of Technology \& Medical Sciences Opposite Oilfed Plant, Indore-Bhopal Road,Sehore (Madhya Pradesh), Pin - 466001

(C) The Authors. Published by Blue Eyes Intelligence Engineering and Sciences Publication (BEIESP). This is an open access article under the CC BY-NC-ND license (http://creativecommons.org/licenses/by-nc-nd/4.0/) the better performance Re at 10,000. Mi-Ae Moon, et.al, [2] this paper have studied the friction loss and heat transfer performances of rib mounted rectangular cooling arrays. This research gives the simulation of different type of sixteen ribs geometry. The pitch to height ratio is confirmed to 10 , width of the rib to hydraulic diameter 0.047. This shoe shape is augmented the heat transfer at the same pressure drop comparing with the square cross section rib. Sang-Hyo Kim, et.al, [3] The Y-type perforated rib is analyzed with the shear forces in simulation software to get the effects of hole diameter, edge distance, transverse rebar diameter on the augmentation of heat transfer.FarzadPourfattah, et.al, [4] The aim of this research work is to analyzed the angle of attack of rib with aluminum nanoparticles for the heat transfer boosting effect.In this it was concluded that, with thepresence ofribs eddy formation is increasedtowards theflow direction results into optimummixing of flow which leads to the heat transfer enhancement. Jinsheng Wang, et.al, [5] In this research rib geometry are changed and analysed like rib stream wise gap distance, width, pitch, inner half rib width angle are analyzed.With the stream size maintained at 0.2 and inner half angle is 450 gives best heat transfer performance and also width gap is promoting to pressure drop but it gives limited heat transfer rate. L. Varshney, et.al, [6] studied twelve types of ribs with different tapered angle are employed for the roughness maintenance of the plate. It was observed that the value of Nussult number and friction factor at constant heat flux. The optimum results found at the Reynolds number 3800 to18000. where the optimum performance index is found at the 1.91 with the reference plate.S. Alfarawi et.al, [7] detremined heat transfer and flow friction for a fully developed turbulent flow in a rectangular duct with its bottom wall ribbed with three different rib geometries such as semi-circular, rectangular and hybrid ribs of the two. The key result of the analysis was the enhancement in the heat transfer is critically influenced by the flow velocity and the turbulence intensity as well as the rib pitch to height ratio (p/e). Alessandro Salvagni, et.al, [8] studied the bottom duct wall, ribbed by flow-normal, equally-distanced square-sectioned ribs, was uniformly heated except for the ribs with an imposed constant heat flux. The outcome for the study was well-resolved LES gave some new insight into the rotation effects on flow and heat transfer, providing information that were not easily accessible to experiments. Nianben Zheng, et.al, [9] studied the heat transfer and friction loss performances of rib-roughened rectangular cooling channels having a variety of cross-sectional rib shapes were analyzed using three-dimensional Reynolds-averaged Navier- Stokes equations. 
The Reynolds stress model was used with the pressure strain model to analyse the turbulence. The computational results for the area-averaged Nusselt number were validated by comparison with the experimental data under the same conditions. The new shoe-shaped rib design showed the best heat transfer performance with a pressure drop similar to that of the square rib.T. Alam,et.al, [10]investigated the application of conical protrusion ribs roughness on the absorber plate of solar air heater duct which showed effective enhancement of the heat transfer rate irrespective of pressure drop penalty. It was observed that The values of friction factor decrease continuously with increasing the relative pitch ratio, which is due to fact that higher values of relative pitch attributed to low resistance offer to flow.

The main inference revealed that rib Shape, pitch, attack angle, height to pitch ratio, arrangement, stream wise gap, etc parameters affect the performance of the rib considerately. Therefore attempt is made in order to optimize the best thermal performance in case of the new stepped shoe shaped ribs.

\section{PROPOSED METHODOLOGY}

\section{a. BLOCK DIAGRAM}

The cross section of experimental air domain is maintained with rectangular shape of dimensions of $180 \mathrm{~mm} \times 290 \mathrm{~mm}$ and the length of it is $2.7 \mathrm{~m}$. In this stepped shoe shape rib mounted plate is placed for the analysis of heat transfer enhancement. The study and analysis is done with simulation software with considering the $\mathrm{K}-\xi$ model. Where the dimensional turbulent velocity is developed by the simulating software and more precise study and analysis can be done respect to it.

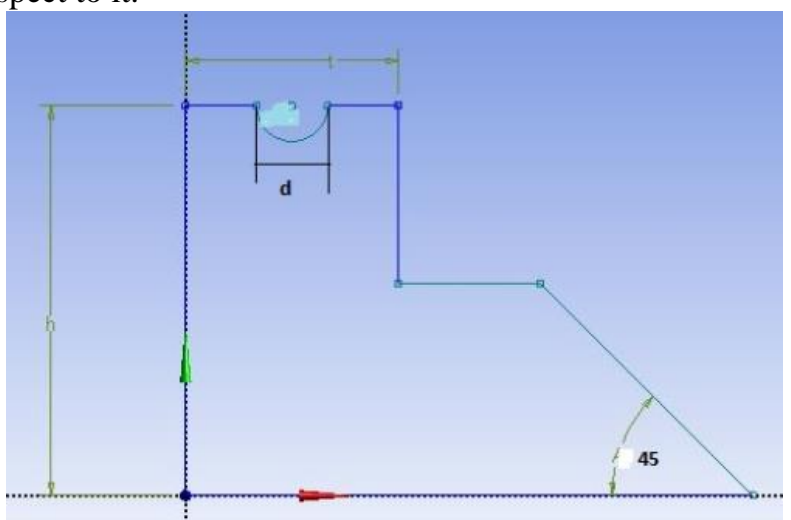

Fig.1. Block Diagram of Grooved Stepped Shoe Shaped Rib

This paper analyses the four main and modified shapes such as flat pate, shoe shape, stepped shoe shape and stepped grooved shoe shape rib. In order to develop turbulence variation in the rib height, rib thickness, groove diameter, is done as per Table I. The simulation study and analysis is carried out with the temperature, pressure and velocity with heat flux of $800 \mathrm{w} / \mathrm{m}^{2}$, velocity is maintained is $2 \mathrm{~m} / \mathrm{s}$.

Table- I:.Parameters under Consideration

\begin{tabular}{|c|c|c|c|c|c|c|}
\hline $\begin{array}{c}\text { Sr. } \\
\text { No }\end{array}$ & Geometry & $\begin{array}{c}\text { Thick } \\
\text { ness } \\
(\mathbf{m m})\end{array}$ & $\begin{array}{c}\text { Height } \\
(\mathbf{m m})\end{array}$ & $\begin{array}{c}\text { Groove } \\
\text { Dia } \\
(\mathbf{m m})\end{array}$ & $\mathbf{h} / \mathbf{t}$ & $\mathbf{d} / \mathbf{t}$ \\
\hline 1 & Flat plate & - & - & - & - & - \\
\hline
\end{tabular}

\begin{tabular}{|c|c|c|c|c|c|c|}
2 & $\begin{array}{c}\text { Simple } \\
\text { Shoe Shape }\end{array}$ & 10 & 20 & - & 2 & - \\
\hline 3 & $\begin{array}{c}\text { Stepped } \\
\text { Shoe Shape }\end{array}$ & 5 & 20 & - & 4 & - \\
\hline 4 & $\begin{array}{c}\text { Stepped } \\
\text { Shoe Shape }\end{array}$ & 4 & 20 & - & 5 & - \\
\hline 5 & $\begin{array}{c}\text { Stepped } \\
\text { Shoe Shape }\end{array}$ & 5 & 15 & - & 3 & - \\
\hline 6 & $\begin{array}{c}\text { Stepped } \\
\text { Shoe Shape }\end{array}$ & 5 & 20 & - & 4 & - \\
\hline 7 & $\begin{array}{c}\text { Stepped } \\
\text { Shoe Shape }\end{array}$ & 5 & 22 & - & 4. & - \\
\hline 8 & $\begin{array}{c}\text { Stepped } \\
\text { Shoe Shape }\end{array}$ & 5 & 20 & 2 & 4 & $\begin{array}{c}0 \\
4\end{array}$ \\
\hline 9 & $\begin{array}{c}\text { Stepped } \\
\text { Shoe Shape }\end{array}$ & 5 & 20 & 3 & 4 & $\begin{array}{c}0 . \\
6\end{array}$ \\
\hline 10 & $\begin{array}{c}\text { Stepped } \\
\text { Shoe Shape }\end{array}$ & 5 & 20 & 4 & 4 & $\begin{array}{c}0 \\
8\end{array}$ \\
\hline 11 & $\begin{array}{c}\text { Truncation } \\
\text { gap 10 mm }\end{array}$ & 5 & 20 & 3 & 4 & $\begin{array}{c}0 . \\
6\end{array}$ \\
\hline 12 & $\begin{array}{c}\text { Truncation } \\
\text { gap 15 mm }\end{array}$ & 5 & 20 & 3 & 4 & $\begin{array}{c}0 . \\
6\end{array}$ \\
\hline 13 & $\begin{array}{c}\text { Truncation } \\
\text { gap 20 mm }\end{array}$ & 5 & 20 & 3 & 4 & $\begin{array}{c}0 . \\
6\end{array}$ \\
\hline 14 & $\begin{array}{c}\text { Truncation } \\
\text { gap 25 mm }\end{array}$ & 5 & 20 & 3 & 4 & $\begin{array}{c}0 . \\
6\end{array}$ \\
\hline
\end{tabular}

\section{A. Analysis for flat plate}

It very important to analyze the heat transfer characteristics of the flat plate to have the reference value to the further work. We have the $4 \mathrm{~mm}$ thick $800 \mathrm{~mm} \times 150 \mathrm{~mm}$ flat plate is simulated as per the given conditions of input with heat flux of $800 \mathrm{w} / \mathrm{m}^{2}$ velocity is $2 \mathrm{~m} / \mathrm{sec}$.

Temperature profile is shown with the streamline indicates that, there is no turbulence generated on the plate, hence there is no turbulence heat transfer from the same flat plate model. Also boundary layer formed on the flat plate which can be differentiatedwith the colour on the fig. 2. Also the temperature absorbed by the working medium i.e air is goes decreases as the gong towards the vertical direction. This is due to no extra efforts are gaining by the heat transfer process. The average temperature of plate is $354 \mathrm{~K}$.

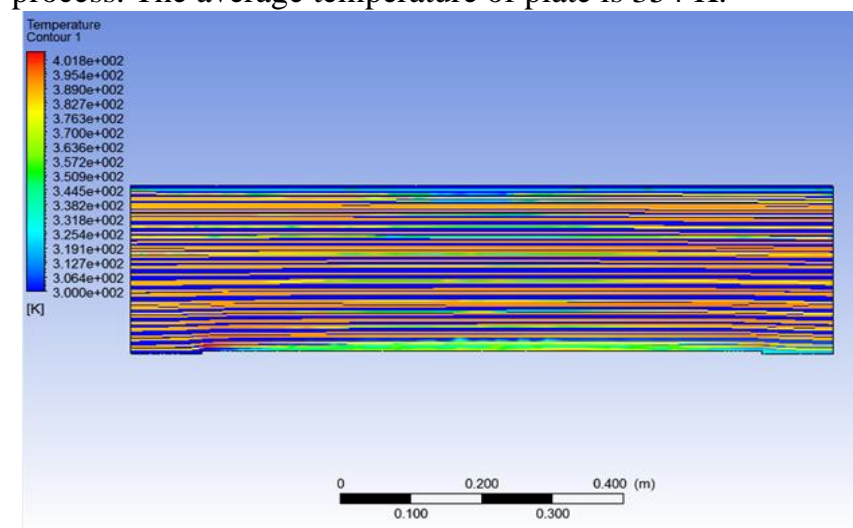

Fig. 2 Temperature contour for flat plate.

Pressure contour showed by the fig. 3 , as there is flat surface for the air flow no pressure generation can be observed in the region belongs to flat plate and the further hence the pressure drop is measured at this stage is $5 \mathrm{~Pa}$. 


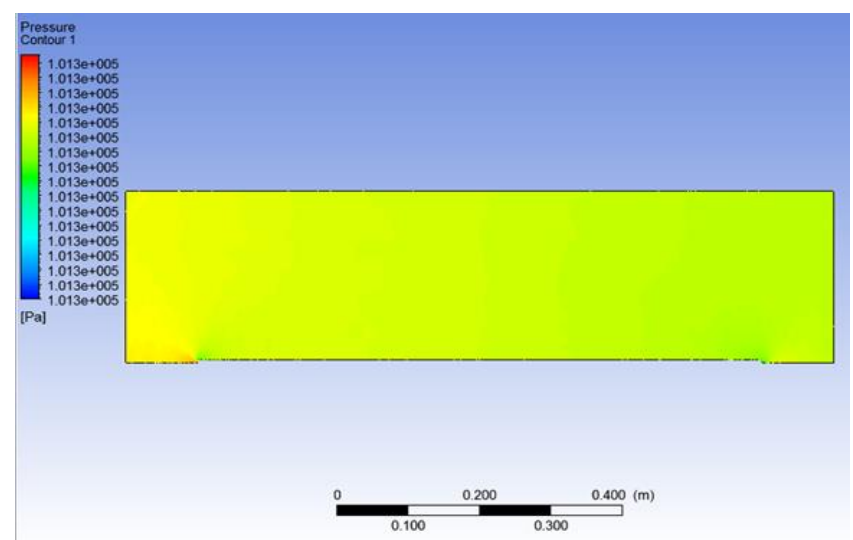

Fig. 3 Pressure contour flat plate.

From the velocity contour (Fig 4) the it shows there is no any turbulence and vortex generation at the region above the flat plate, hence the heat transfer coefficient obtained is lower, ultimately we have the scope to improve this parameter to get the maximum augmentation of the heat transfer. Nussult number from the simulation is 95.781 and heat transfer coefficient is $14.81 \mathrm{~W} / \mathrm{mK}$.



Fig. 4 Velocity contour flat plate.

B. Analysis based on geometrical variation of shoe shape Ribs

Shoe shape rib of height $20 \mathrm{~mm}$ and width $20 \mathrm{~mm}$ is placed on the flat plate over the width of $150 \mathrm{~mm}$. This plate is place with above mentioned condition and the simulation is done with temperature, velocity and pressure are discussed below as per point 2 to 10 (table I).It Shows the turbulence generated by the shoe shape on the plate profile which can be augment the heat transfer from the plate as turbulence created by the shoe shape rib. Pressure drop can be observed to the pressure tile which gives the enhancement effect to the heat transfer. Similar type of simulation is done with the stepped shoe shape rib mounted plate, the profiles are discussed. Shoe shape rib creates the pressure at the side the wall of rib as this pressurized air is travelled over the head of the rib it converts in to velocity and get boosted up.

I] Temperature distribution of the shoe shape rib

Various temperature profile for the different geometrical shapes of shoe shaped ribs are shown in figures.

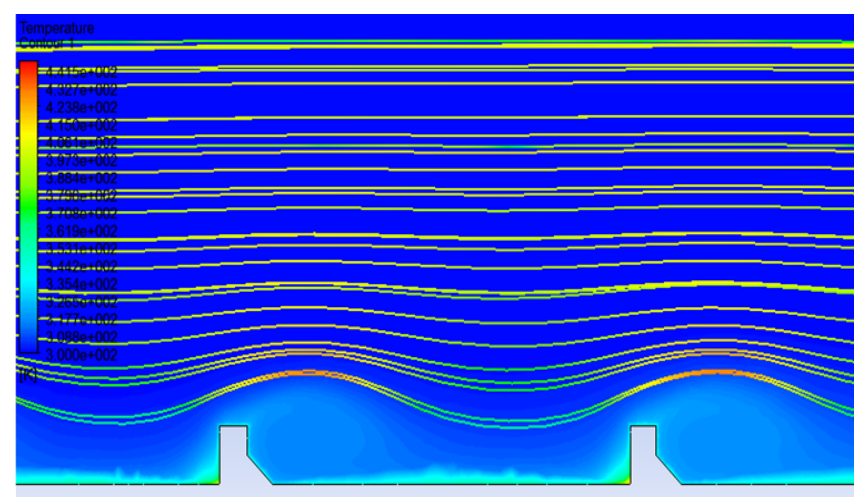

Fig. 5.Temperature profile of shoe shape rib

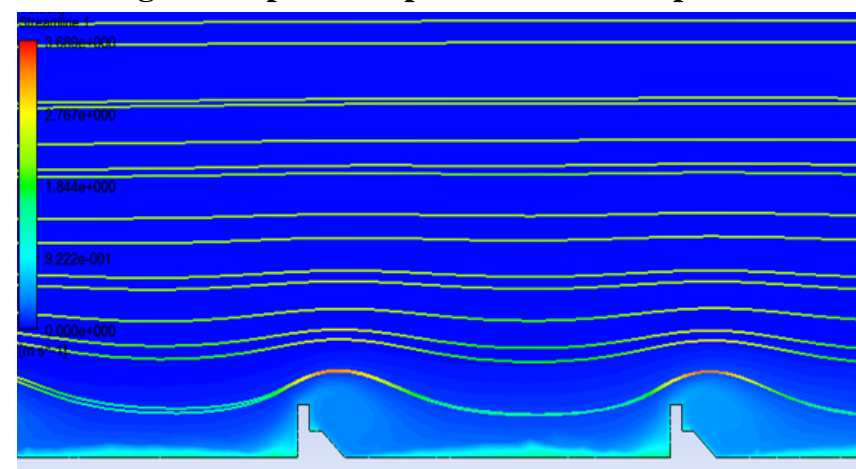

Fig. 6 Temperature profile of stepped shoe shape rib.

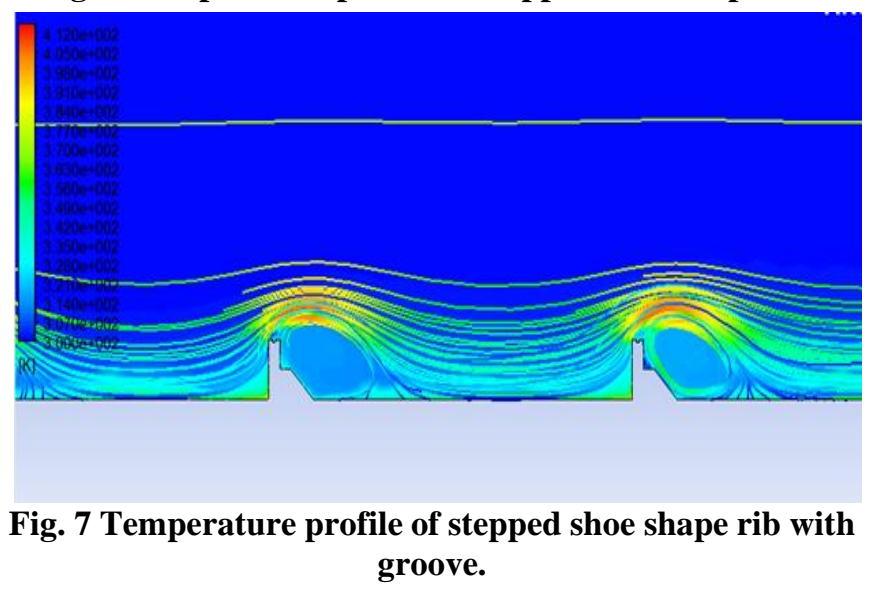

Fig. 5 to 7 shows temperature contours for shoe shaped rib, stepped shoe shape rib, and stepped shoe shape rib with groove. The average surface temperature obtained in above cases is $342.702 \mathrm{~K}, 339.958 \mathrm{~K}, 336.915 \mathrm{~K}$ respectively. Stepped shoe shape ribs with groove gives the smallestaverage temperature of the surface with above geometries which result in higher heat transfer coefficient across the plate. From the above mentioned result it can be attributed that geometrical variation have significant effect on the average surface temperature of heater plate. Also the streamline shows the vortex generated with respect to the temperature distribution over the plate surface.

Shoe shape ribs gives the pressure drop of $8 \mathrm{~Pa}$ and Nussult number is 121.13 and heat transfer coefficient is 18.7345 $\mathrm{W} / \mathrm{m}^{2} \mathrm{~K}$. which shows the better heat transfer performance with respect to flat plate. 


\section{II] Velocity distribution of the shoe shape rib}

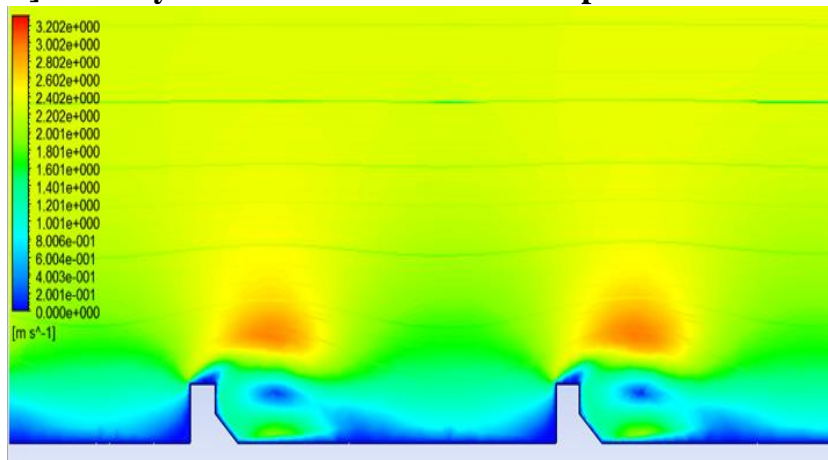

Fig. 8. Velocity profile of shoe shape rib

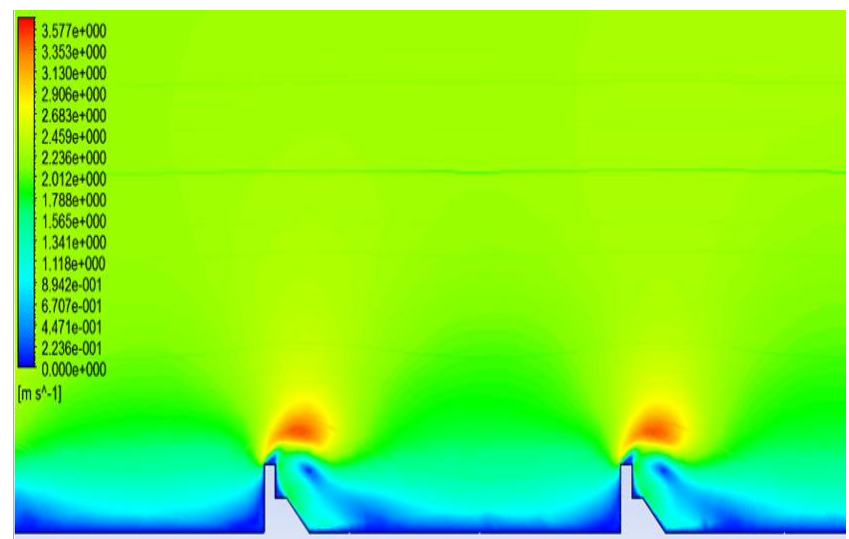

Fig. 9 Velocity profile of stepped shoe shape rib.

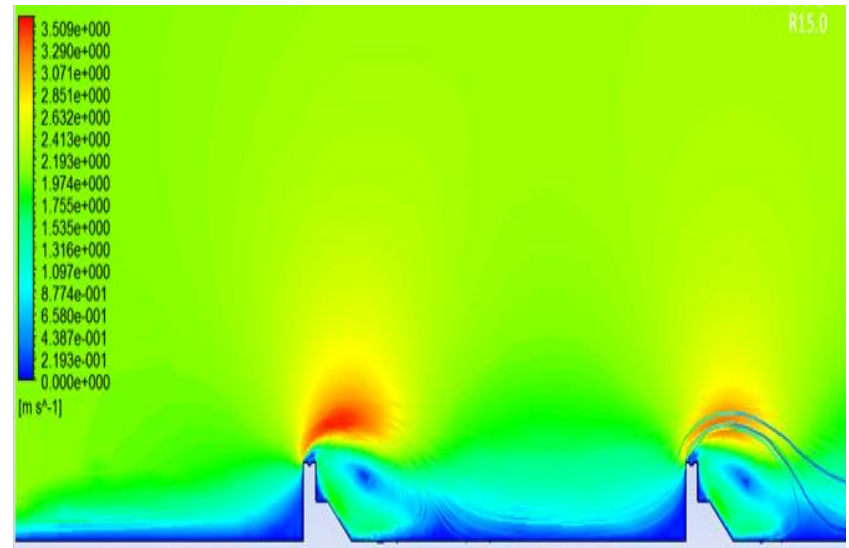

Fig. 10 Velocity profile of stepped shoe shape rib with groove.

Fig. 8 to 10it is observed there is vortex generation after the ribs and subsequent reattachment of it further downstream between the two ribs. Thisphenomenon is responsible for increase in heat transfer and increase in pressure drop. In case of filleted shoe ribs vortex generation is large as compared to shoea rib which leads to significant increase in heat transfer, while in case of stepped shoe ribs there is secondary vortex generation along with primary vortex which leads to localized hot spots in groove due secondary vortex and decreases the heat transfer. This gives the more groove shape on the rib head gives the significant increase in Nussult number leads to have more heat transfer coefficient.

\section{III] Pressure distribution of shoe Shape}

Shoe shape gives the best energy transfer as the pressure developed by the ribs converted in to the velocity which gives the augmentation in the heat transfer. Modification in the shoe shape gives thepressuredrop $8 \mathrm{~Pa}, 9 \mathrm{~Pa}, 8 \mathrm{~Pa}$. This pressure drop gives the friction factor enhancement on the increase in the same.

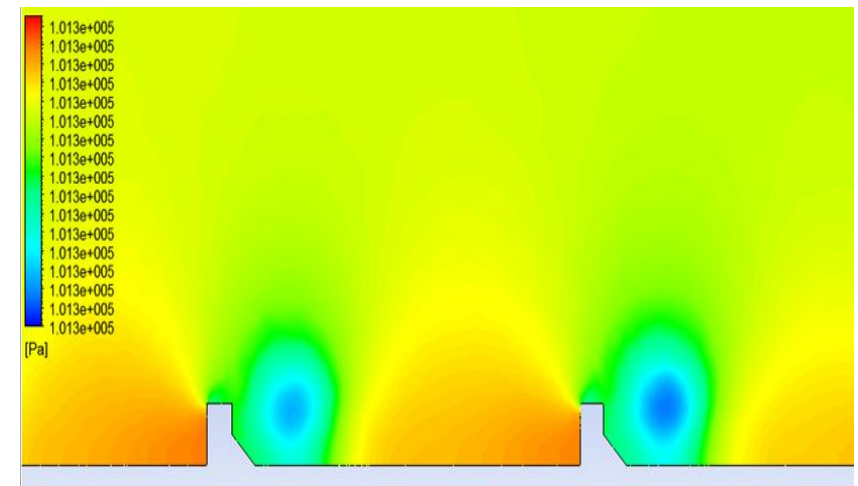

Fig. 11 Pressure profile shoe shape rib

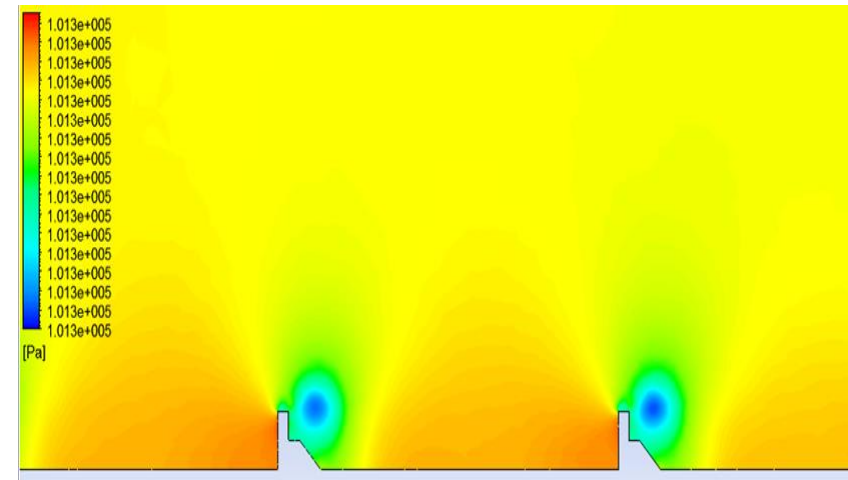

Fig. 12 Pressure profile of stepped shoe shape rib.

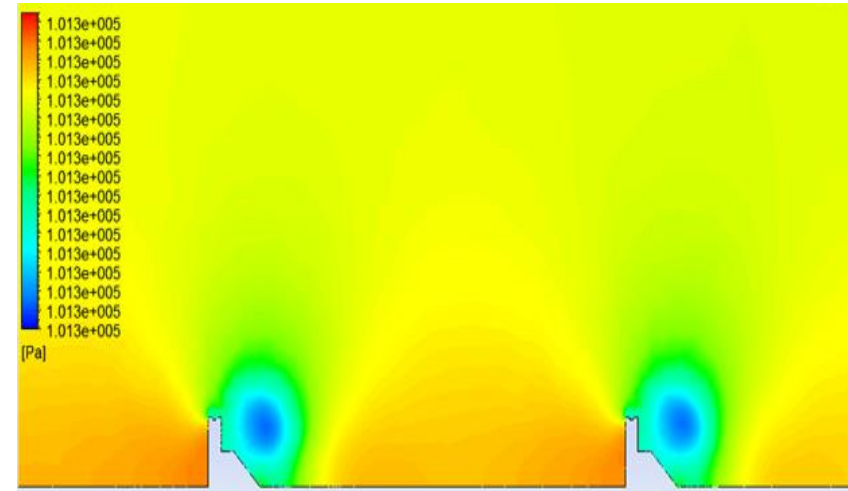

Fig. 13 pressure profile of stepped rib with groove C. Data Reduction

\section{1] Average surface temperature (Ts)}

It can be taken from the obtained temperature plots

\section{2] Nusselt number}

The Nusselt number is a measure of the convective heat transfer occurring at the surface and is defined as $\mathrm{hd} / \mathrm{k}$, where $h$ is the convective heat transfer coefficient, $d$ is the diameter of the tube and $\mathrm{k}$ is the thermal conductivity.

$\mathrm{Nu}=\frac{\mathrm{qD}_{\mathrm{h}}}{\mathrm{k}\left(\mathrm{T}_{\mathrm{S}}-\mathrm{T}_{\mathrm{O}}\right)}$

\section{3] Friction factor}

The friction factor is a measure of head loss or pumping power. 
$\mathrm{f}=\left(\frac{\Delta \mathrm{P}}{\frac{1}{2} \rho \mathrm{U}_{\mathrm{m}}^{2}}\right) \frac{\mathrm{D}_{\mathrm{h}}}{\mathrm{L}}(2)$

4] Thermal enhancement factor

The thermal enhancement factor is defined as the ratio of the heat transfer enhancement ratio to the friction factor ratio. This parameter is also used to compare different passive techniques and enables a comparison of two different methods for the same pressure drop.

$\mathrm{TEF}=\frac{\overline{\mathrm{Nu}} / \overline{\mathrm{Nu}}_{\mathrm{o}}}{\left(\overline{\mathrm{f}} / \overline{\mathrm{f}}_{\mathrm{o}}\right)^{\frac{1}{3}}}$

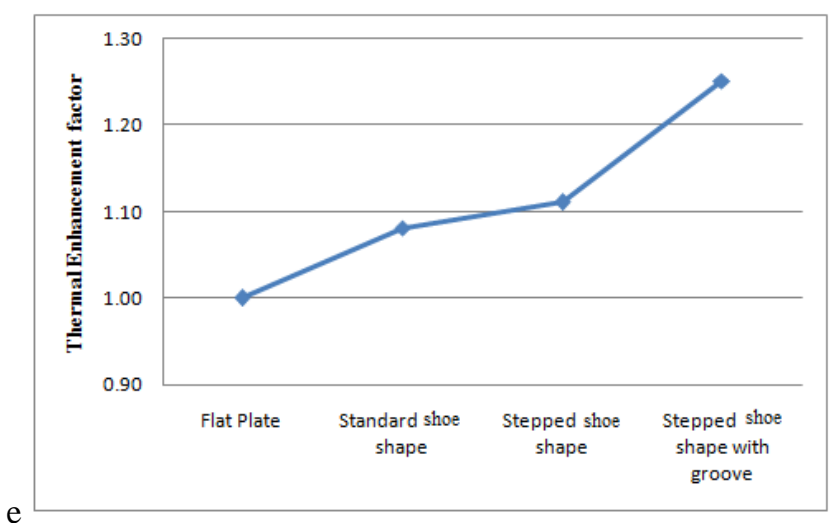

Fig. 14 Thermal Enhancement Factor

Fig. 14 shows the thermal enhancement factor that shows the standard shoe shape gives the 1.08 as of the stepped modified shoe shape gives the further augmentation to 1.11 . Stepped shoe shape with the groove on the head of the rib is states the 1.25 which gives the $15 \%$ thermal enhancement over the standard shoe shape rib. As the best indication given from the stepped shoe shape with groove with the temperature and velocity with best pressure drop where observed.

D. Modification of the rib spacing in truncation with staggered arrangement

Thermal enhancement from the plate can be depend upon the arrangement of the rib that the truncation staggered arrangement gives the further enhancement of heat transfer is calculated at the centered arrangement with the different gaps were analyzed. The new arrangement consists of ribs having pitch $\mathrm{P} / 2$. A slight gap is provided in between to check if it is possible to get better thermal characteristics. Three gaps provided are $5 \mathrm{~mm}, 10 \mathrm{~mm}$ and $15 \mathrm{~mm}$ respectively. CFD analysis is performed for this new arrangement and truncation gap and results are obtained to investigate the modifications done in the geometry. The air velocity is $2 \mathrm{~m} / \mathrm{s}$ and the heat flux provided is $800 \mathrm{~W} / \mathrm{m} 2$.

I] Temperature and streamline profile of truncation with staggered arrangement of the ribs

Temperature and streamline distribution of the truncation with staggered arrangement of the rib with truncation gap of $10 \mathrm{~mm}, 15 \mathrm{~mm}$ and $20 \mathrm{~mm}$ are shown in fig 15 t0 17 respectively.The truncation gap helps to developed turbulence. It is observed that the highest value of heat transfer is possible in case of truncation than that of inline , staggered arrangement. With this simulation analysis it is observed that that the best heat transfer coefficient to the 20 $\mathrm{mm}$ truncation gap $25.2797 \mathrm{w} / \mathrm{m}^{2} \mathrm{k}$ for the temperature of $331.646 \mathrm{k}$

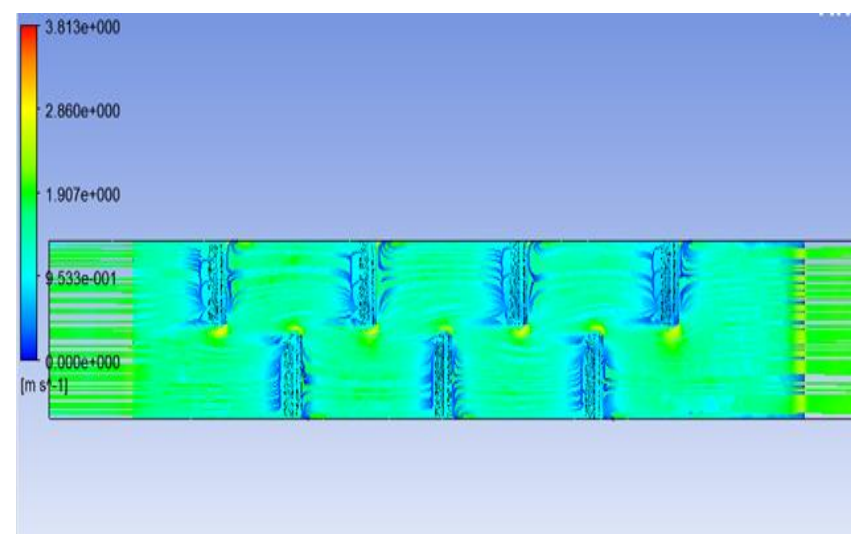

Fig. 15 Temperature and streamline profile for $10 \mathrm{~mm}$ truncation gap

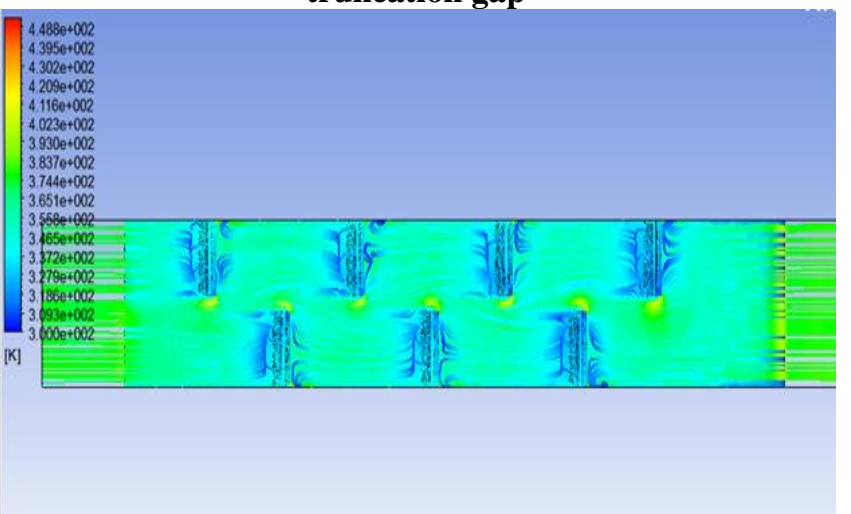

Fig. 16 Temperature and streamline profile for $15 \mathrm{~mm}$ truncation gap

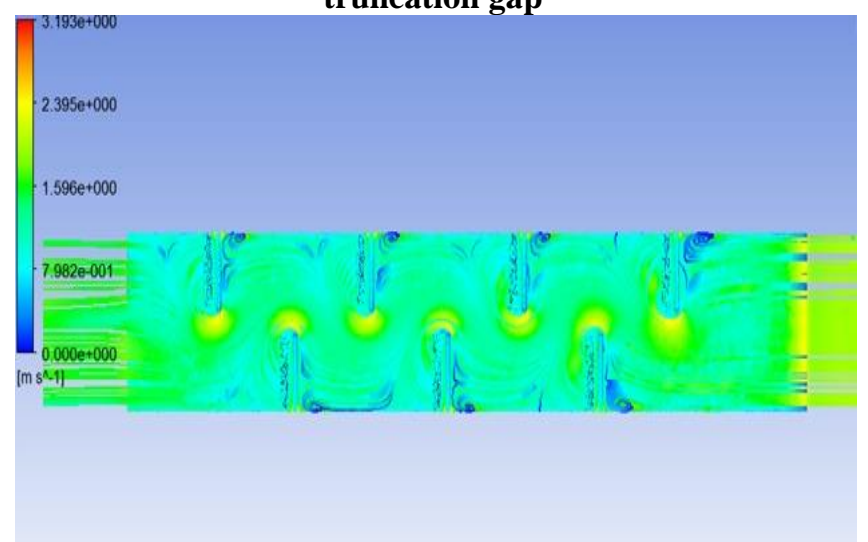

Fig. 17 Temperature and streamline profile for $20 \mathrm{~mm}$ truncation gap

.II] Pressure profiles for various truncation gaps

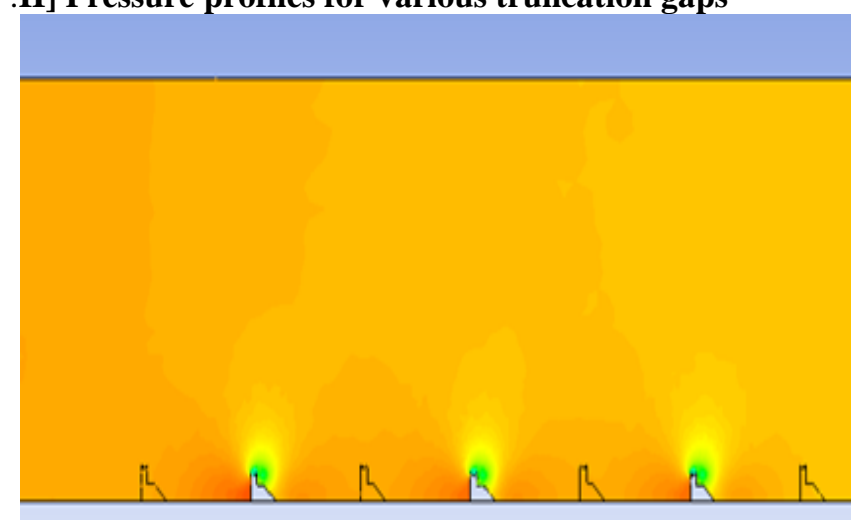

Fig. 18 Pressure profile for the truncation gap $10 \mathrm{~mm}$.

Published By:

Blue Eyes Intelligence Engineering

\& Sciences Publication 


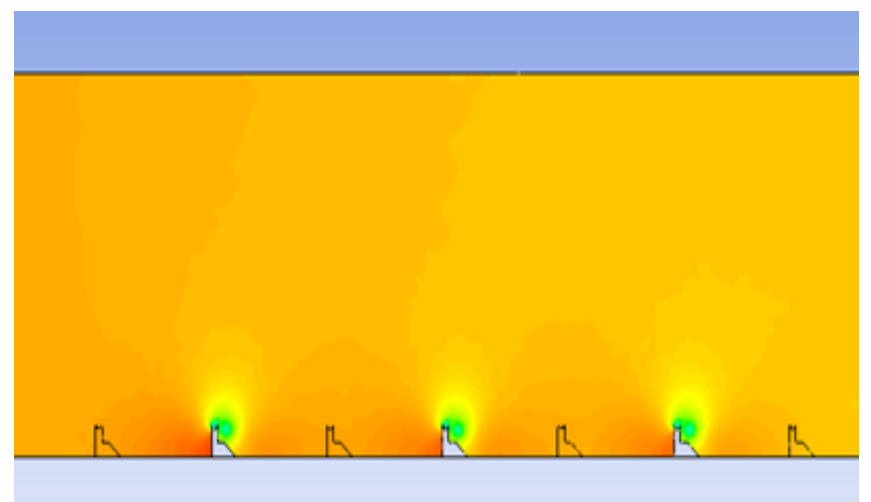

Fig. 19 Pressure profile for the truncation gap $15 \mathrm{~mm}$.

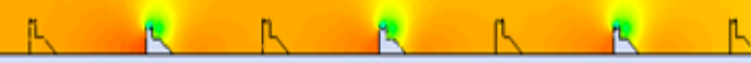

Fig. 20 Pressure profile for the truncation gap $20 \mathrm{~mm}$

Fig.18 to 20 shows pressure contours for filleted shoe shaped ribs with mid-truncation of $10 \mathrm{~mm}, 15 \mathrm{~mm}$ and $20 \mathrm{~mm}$. The pressure difference obtained in above cases is almost similar with pressure drop being of $14 \mathrm{~Pa}$ respectively.

\section{RESULT ANALYSIS}

\section{A. Effect of geometry variation on Nusselt Number}

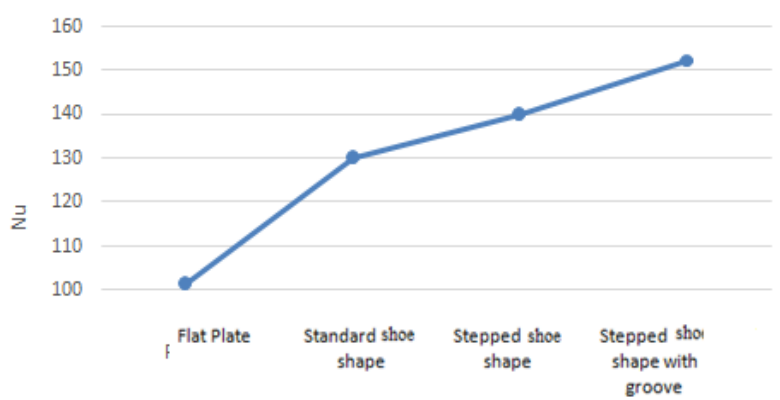

Fig. 21 Effect of Variation in Geometry on Nusselt Number

The effect of geometry variation on Nusselt number is shown in above figure 21, The turbulent heat transfer from the stepped shoe rib gives the augmentation in heat transfer for the step thickness of $5 \mathrm{~mm}$. This modified stepped shoe shape rib gives increased heat transfer properties with respect to the normal shoe shape rib. The trend shows that the Nussult number increases with modification in the rib shapes over the flat plate. Further increase in the turbulence in the path of the stream can be achieved by providing groove, which is leads to increase the Nussult number. The increment of $50.37 \%$ in Nusselt number is achieved forthe grooved boot shape rib over the flat plate.

\section{B. Effect of Truncation gap variation on Nusselt Number}

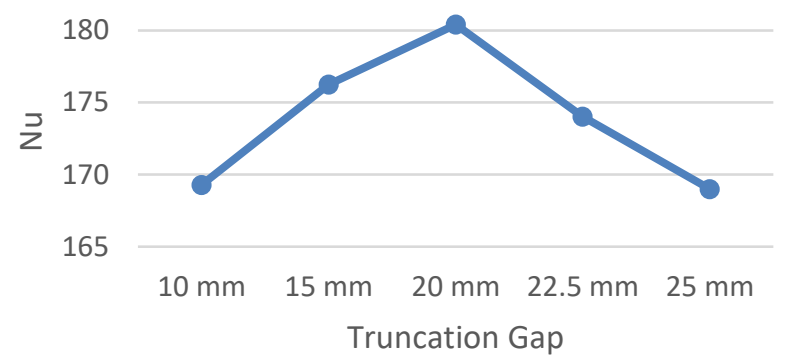

Fig. 22 Effect of truncation gap on Nusselt Number The trends showing effect of variation on the truncation gap on the Nussult number. In this case the truncation gaps are varied such as $10 \mathrm{~mm}, 15 \mathrm{~mm}, 20 \mathrm{~mm}, 22.5 \mathrm{~mm}, 25 \mathrm{~mm}$. Trend gives the increment in the Nussult number from the 10 $\mathrm{mm}$ to $20 \mathrm{~mm}$, further the Nusselt number drops due to non contact of air on the ribs. The highestNussult number gives at the truncation gap of $20 \mathrm{~mm}$ is 180.40 . In this turbulence created between the truncation .

\section{Effect of Truncation gap variation on Enhancement} factor

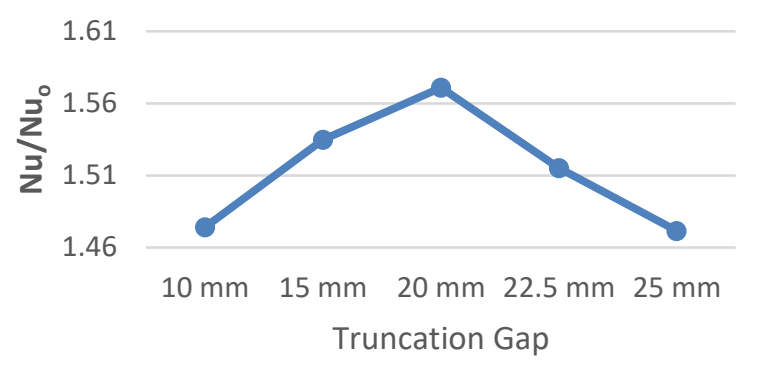

Fig. 23 Effect of truncation gap on Enhancement factor

Truncation with staggered arrangement gives the best results in the heat transfer enhancement. Providing staggered arrangement truncation gap reduces the surface temperature gradually till $20 \mathrm{~mm}$ and gives increased value of surface temperature further increase in truncation gap. It is observed that there is increasing trend in $\mathrm{Nu} / \mathrm{Nuo}$ ratio for increasing truncation gap up to $20 \mathrm{~mm}$, beyond which there is decreases in $\mathrm{Nu} / \mathrm{Nuo}$ ratio. $\mathrm{Nu} / \mathrm{Nuo}$ ratio increases from 1.47 for $10 \mathrm{~mm}$ to 1.57 for $20 \mathrm{~mm}$ and then decreases to 1.47 for $25 \mathrm{~mm}$

\section{Effect of Truncation gap variation on Thermal Enhancement factor}

The thermal enhancement factor includes the effect of friction factor also. Therefore it gives clear indication of increase in heat transfer over change in pressure drop due to turbulence. The increasing trend of thermal enhancement factoris observed for increasing truncation gap up to $20 \mathrm{~mm}$, beyond which there is decreases in TEF. This is due to the combination of mixing of the flow which leads to the generation of turbulence and augmentation of the Nussult number. The highest value of thermal enhancement factor is 1.33 at $20 \mathrm{~mm}$ truncation gap. The increase in truncation gap beyond $20 \mathrm{~mm}$ reduces the thermal enhancement factor. 


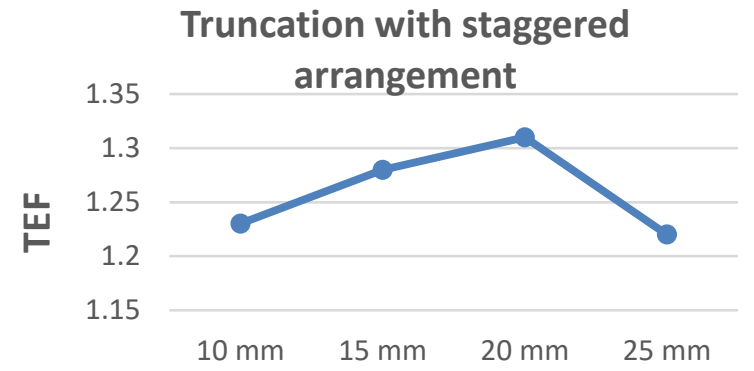

Fig. 24Effect of truncation gap on Thermal Enhancement factor

\section{RESULT TABLE}

Table- II: Result Table

\begin{tabular}{|c|c|c|c|c|}
\hline $\begin{array}{l}\text { Sr. } \\
\text { No }\end{array}$ & Geometry & Nu & $\begin{array}{c}\mathrm{Nu} / \mathrm{N} \\
\text { uo }\end{array}$ & TEF \\
\hline 1 & Flat plate & 100 & 1 & 1 \\
\hline 2 & Simple Shoe Shape & 130 & 1.21 & 1.18 \\
\hline \multicolumn{5}{|c|}{ Stepped Shoe Shape } \\
\hline 3 & $\mathrm{~h} / \mathrm{t}=4$ & 148.57 & 1.42 & 1.27 \\
\hline 4 & $\mathrm{~h} / \mathrm{t}=5$ & 135.67 & 1.43 & 1.20 \\
\hline 5 & $\mathrm{~h} / \mathrm{t}=3$ & 129.46 & 1.32 & 1.18 \\
\hline 6 & $\mathrm{~h} / \mathrm{t}=4.4$ & 146.45 & 1.39 & 1.23 \\
\hline 7 & $\begin{array}{c}\mathrm{h} / \mathrm{t}=4 \\
\mathrm{~d} / \mathrm{t}=0.4\end{array}$ & 160.22 & 1.42 & 1.22 \\
\hline 8 & $\begin{array}{c}\mathrm{h} / \mathrm{t}=4 \\
\mathrm{~d} / \mathrm{t}=0.6\end{array}$ & 167.21 & 1.46 & 1.25 \\
\hline 9 & $\begin{array}{c}\mathrm{h} / \mathrm{t}=4 \\
\mathrm{~d} / \mathrm{t}=0.8\end{array}$ & 162.37 & 1.43 & 1.21 \\
\hline 10 & $\begin{array}{c}\text { Truncation gap } 10 \mathrm{~mm} \\
\mathrm{~h} / \mathrm{t}=4 \\
\mathrm{~d} / \mathrm{t}=0.6\end{array}$ & 169.2 & 1.48 & 1.23 \\
\hline 11 & $\begin{array}{c}\text { Truncation gap } 15 \mathrm{~mm} \\
\mathrm{~h} / \mathrm{t}=4 \\
\mathrm{~d} / \mathrm{t}=0.6\end{array}$ & 176.41 & 1.54 & 1.26 \\
\hline 12 & $\begin{array}{c}\text { Truncation gap } 20 \mathrm{~mm} \\
\mathrm{~h} / \mathrm{t}=4 \\
\mathrm{~d} / \mathrm{t}=0.6\end{array}$ & 180.41 & 1.58 & 1.33 \\
\hline 13 & $\begin{array}{c}\text { Truncation gap } 25 \mathrm{~mm} \\
\mathrm{~h} / \mathrm{t}=4 \\
\mathrm{~d} / \mathrm{t}=0.6\end{array}$ & 168.35 & 1.47 & 1.22 \\
\hline
\end{tabular}

Table II represents the key results of geometry variation of stepped grooved shoe shape rib in the form of Nusselt Number $(\mathrm{Nu})$, Enhancement factor $\left(\mathrm{Nu} / \mathrm{Nu}_{0}\right)$ and thermal enhancement factor (TEF).

The geometrical variation consider are flat plate (1), simple shoe shape ribs (2), Stepped Shoe shape ribs (3-6), Stepped grooved shoe shaped ribs (7-9) and truncation gap variation 10 to $25 \mathrm{~mm}$ (10-13). The thermal enhancement factor (TEF) is main deciding parameter as it includes effect of friction factor as per equation (3). It is observed that the magnitude of TEF is lowest for the simple shoe shape ribs (1.18). In case of stepped shoe shape ribs due to the step the optimum result obtained at $h / t=4(T E F=1.27)$. The TEF increases by provision of groove on the top of the rib. The highest value of TEF achieved at $\mathrm{d} / \mathrm{t}=0.6$ is 1.25 .
The enhancement in heat transfer is due to creation of turbulence on provision of grooves on ribs top. The truncation gap plays main role in augmentation of heat transfer and lowering pressure drop. The optimum value of thermal enhancement factor is 1.33 at $20 \mathrm{~mm}$ truncation gap.

\section{CONCLUSIONS}

1. The new Stepped Grooved shoe shape rib can be considered as an heat transfer augmentation tool. The shoe shape thickness and groove diameter plays main role in heat transfer increment.

2. For stepped with grooved shoe shape rib heat transfer increases with turbulence which gives the heat transfer enhancement with respect to the flat plate.

3. Varying the thickness of the stepped shoe shape rib has significant effect on heat transfer rate. It gives highest results for the stepped boot shape at $5 \mathrm{~mm}$ head thickness of the stepped rib.

4. As the thickness of the shoe head is modified to $5 \mathrm{~mm}$, Nussult number and heat transfer augmentation observed numerically in thermal enhancement factor to 1.11.It can be concluded that the head thickness of the stepped shoe shape rib (up to $5 \mathrm{~mm}$ ) helped in better heat transfer characteristics.

5. Providing the groove on the head of the stepped shape of the rib gives better turbulence in the path of the flow. Modifying the diameter of the groove $(3 \mathrm{~mm})$ gives the best enhancement in the heat transfer. Thermal enhancement factor also gives augmentation to the stepped with groove boot shape rib of 1.25.

6. Providing staggered arrangement with truncation gap of $20 \mathrm{~mm}$ gives the highest value of thermal enhancement factor of 1.33 due to best mixing of the flow. Nusselt and thermal enhancement factor to optimized arrangement is 180.40 .

\section{REFERENCES}

1. Deep Singh Thakur, Performance Evaluation of Solar Air Heater With Novel Hyperbolic Rib Geometry, Int. J. of Renewable Energy, 105 (2017), pp 786-797

2. Mi-Ae Moon, Min-Jung Park, Kwang-Yong Kim, Evaluation of heat transfer performances of various rib shapes, International Journal of Heat and Mass Transfer 71 (2014) pp 275-284

3. Sang-Hyo Kim, "Generalized formulation for shear resistance on Y-type profound rib shear connectors" 2016

4. FarzadPourfattah, "The numerical investigation of angle of attack of inclined rectangular rib on the turbulent heat transfer of Water-Al2O3 NanoFluid in a tube” Int. J. of Mechanical Sciences ,(17), 2017.

5. Jinsheng Wang, Numerical investigation of heat transfer and fluid flow in a rotating rectangular channel with variously-shaped discrete ribs, Int. J. of App. Thermal Engineering, 129 (2018) 1369-1381.

6. L. Varshney, A.D. Gupta, Performance prediction for solar air heater having rectangular sectioned tapered rib roughness using CFD, 4 (2017) pp 122-132.

7. S. Alfarawi , S.A. Abdel-Moneim, A. Bodalal, Experimental investigations of heat transfer enhancement from rectangular duct roughened by hybrid ribs, Int. J. of Thermal Sciences, 118 (2017) 123-138

8. Alessandro Salvagni "Numerical investigation of heat transfer and fluid flow in a rotating rectangular channel with variously-shaped discrete ribs" Int. J. of Applied Thermal Engineering, (17), 2017

9. N. Zheng, P.Liu, F.Shan, Z.Liu, W.Liu,Effects of rib arrangements on the flow pattern and heat transfer in an internally ribbed heat exchanger tube, Int. J. Therm. Sci, 101 (2016) 93-105. 
10. TabishAlam, Man-HoeKim, Heat transfer enhancement in solar air heater duct with conical protrusion roughness ribs, J. of Applied Thermal Engineering, 126(2017)458-469

\section{AUTHORS PROFILE}

Mr. Sameer Y. Bhosale has completed ME in Heat Power Engineering in First class with distinction. His research area of interest is Heat transfer. He has published $50+$ research papers in reputed national and international journals

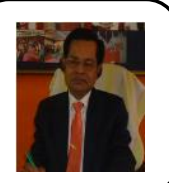

Prof.Dr.G.R.Selokar is Registrar at Sri SatyaSai University of Technology \& Medical Sciences Opposite Oilfed Plant, Indore-Bhopal Road,Sehore (Madhya Pradesh), Pin -466001 . 